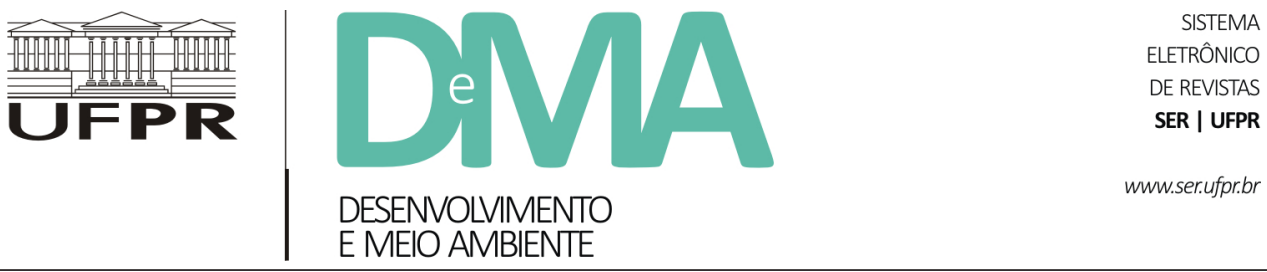

\title{
Prevenção de desastres de origem hídrica no Brasil: avaliação do nível de implementação do Quadro de Ação de Hyogo
}

\section{Waterborne Disaster Prevention in Brazil: Assessing the Level of Implementation of the Hyogo Framework for Action}

\author{
Maria do Socorro Lima Castello BRANCO ${ }^{1}$, Carlos Hiroo SAITO2* \\ ${ }^{1}$ Agência Nacional de Águas (ANA), Brasília, DF, Brasil \\ ${ }^{2}$ Centro de Desenvolvimento Sustentável (CDS), Universidade de Brasília (UnB), Brasília, DF, Brasil. \\ *E-mail de contato: carlos.h.saito@hotmail.com
}

Artigo recebido em 3 de outubro de 2016, versão final aceita em 11 de setembro de 2017.

RESUMO: A preocupação com o aumento dos desastres naturais de origem hidrológica tem crescido no mundo, levando à aprovação da Estratégia Internacional para a Redução de Desastres - EIRD pela Assembléia Geral da Organização das Nações Unidas, em dezembro de 1999. Esta estratégia está consolidada no Quadro de Ação de Hyogo 2005-2015 (QAH): construindo a resiliência das nações e comunidades a desastres. Considerase que o QAH é importante para o Brasil enquanto país que vem sofrendo um processo de urbanização acelerado e demonstrado vulnerabilidade de suas cidades aos eventos hidrológicos. Assim, o objetivo desta pesquisa foi avaliar o nível de implementação do QAH pelo Brasil sob o ponto de vista da valorização da prevenção de desastres nas políticas públicas. A metodologia usada foi predominantemente qualitativa, com análise de documentos e entrevistas, à luz de uma estrutura de indicadores de efetividade. Os resultados obtidos demonstraram o avanço obtido pelo país no âmbito da governança e da identificação dos riscos, mas evidenciam a necessidade de gerir o conhecimento adquirido, de reduzir os fatores de risco e de fortalecer a capacidade de resposta.

Palavras-chaves: Hyogo; cidades resilientes; inundações; segurança hídrica.

ABSTRACT: The concern about the increase of natural disasters with hydrological origin has grown worldwide, leading to the approval of the International Strategy for Disaster Reduction - ISDR by the United Nations General Assembly in December 1999. This strategy is consolidated in the Hyogo Framework for Action 2005-2015 (HFA): building the resilience of nations and communities to disasters. We consider HFA important for Brazil as a country that is suffering an accelerated urbanization process and has demonstrated vulnerability of their 
cities to hydrological events. Thus, the aim of this study was to evaluate the level of implementation of the HFA by Brazil focused on the enhancement of disaster prevention in public policy. The applied methodology was predominantly qualitative, based on documents and interviews analysis in a framework of indicators of effectiveness. The results demonstrated the progress the country in governance and risk identification, but stress the need to manage knowledge acquired, to reduce risk factors and strengthen the response capacity

Keywords: Hyogo; resilient cities; floods; water security.

\section{Introdução}

A preocupação com o aumento dos desastres naturais de origem hidrológica tem crescido no mundo, tendo em vista o reconhecimento tanto pela população como pelos gestores governamentais de que o rio que abastece as cidades é o mesmo que as invade, que as vulnerabiliza social, ambiental e economicamente. $\mathrm{O}$ mesmo ocorre com as chuvas. Durante os eventos hidrológicos de aumento de vazão dos rios ou eventos meteorológicos de excesso de chuvas, a água deixa de ser vista como uma dádiva da natureza e passa a ser a responsável pela perda de vidas e pelos prejuízos materiais e de qualidade de vida que provoca: passa a ser vista como fonte de desastres (Santos, 2008; Guimarães et al., 2008; Chiapetti \& Chiapetti, 2011; Kazmierczak \& Cavan, 2011).

A partir do aumento e repetição do número de eventos de desastres, surge a preocupação com a busca de maior proteção contra esses episódios. Dessa preocupação, nasce o conceito de segurança hídrica, que expressa, para além da disponibilidade de água potável em quantidade e qualidade, a garantia de que a água não causará desastres (GWP, 2000; Lautze \& Manthrithilake, 2012).

Concomitantemente à gestação do conceito de segurança hídrica, cresce no interior da Organização das Nações Unidas (ONU) a preocupação com a recorrência de desastres mundo afora, levando sua
Assembleia Geral a aprovar, em dezembro de 1999, a Estratégia Internacional para a Redução de Desastres - EIRD (International Strategy for Disaster Reduction - ISDR). Essa estratégia buscou chamar a atenção das autoridades nacionais e na escala local para a importância da redução do risco de desastres, desenvolvendo comunidades mais resilientes aos riscos naturais.

Para implementar essa estratégia, foi fundado o Escritório das Nações Unidas para a Redução de Risco de Desastres (United National Office Disaster Risk Reduction - UNISDR), bem como foi produzido o documento "Quadro de Ação de Hyogo 2005-2015: construindo a resiliência das nações e comunidades a desastres" (UNISDR, 2007). O Quadro de Ação de Hyogo (QAH) foi aprovado e adotado por 168 membros das Nações Unidas, inclusive o Brasil, em 2005.

De acordo com o Anuário Brasileiro de Desastres Naturais em 2012, elaborado pelo Centro Nacional de Gerenciamento de Riscos e Desastres (CENAD), 7.067.958 de brasileiros $(3,55 \%$ da população) foram vítimas de desastres de origem hidrológica durante o ano de 2012, sendo a inundação a modalidade de desastre de maior impacto no território nacional (Brasil, 2013). Apesar da gravidade do tema, há carência de avaliação das políticas de prevenção de desastres no Brasil, ou do grau de aderência das ações praticadas ao marco referencial QAH que o país aderiu. 
Desse modo, o objetivo do presente trabalho foi avaliar o nível de implementação do Quadro de Ação de Hyogo no que se refere à prevenção e mitigação de desastres no Brasil, sob o ponto de vista da valorização da prevenção de desastres nas políticas públicas.

\section{Procedimentos metodológicos}

Foi realizada uma pesquisa documental no orçamento federal, particularmente no Plano Plurianual (PPA), e na legislação brasileira, contemplando a Lei n. ${ }^{\circ}$ 9.433/1997, Lei n. ${ }^{o}$ 9.795/1999, Lei n. ${ }^{\circ} 12.340 / 2010$, Lei n. ${ }^{\circ} 12.527 / 2011$, Lei n. ${ }^{\circ}$ 12.608/2012, Decreto n. ${ }^{\circ} 7.257 / 2010$, Decreto n. ${ }^{\circ}$ $8.161 / 2013$, Decreto n. ${ }^{\circ} 8.243 / 2014$, Portaria Conjunta . $^{\circ} 148$, de 18 de dezembro de 2013 e Instrução Normativa $n .^{\circ} 1$, de 24 de agosto de 2012, visando compreender os mecanismos institucionais em vigor que afetam as ações de prevenção e mitigação de desastres. Além disso, foram obtidos dados atualizados com o Centro Nacional de Monitoramento e Alertas de Desastres Naturais do Ministério da Ciência, Tecnologia e Inovação (CEMADEN/MCTI), entre eles, as seguintes listagens: os 821 municípios críticos do Plano Nacional de Gestão de Riscos e Resposta a Desastres Naturais, os 286 municípios prioritários para monitoramento pelo CEMADEN, os municípios com ocorrência de desastres naturais de origem hidrológica, separados em inundações, enxurradas e deslizamentos, os municípios que aderiram ao QAH.

Entrevistas semiestruturadas foram conduzidas com 19 atores sociais envolvidos no processo, buscando pontos de vista de representantes de cada um dos órgãos envolvidos no monitoramento e na prevenção de desastres, em posição hierárquica de comando, tal que conduzissem a uma maior compreensão do sistema: a) Secretaria de Planejamento e Investimento do Ministério do Planejamento, Orçamento e Gestão (SPI/MPOG); b) CENAD, vinculado à - Secretaria Nacional de Defesa Civil do Ministério da Integração Nacional (SEDEC/MI); c) Secretaria Nacional de Acessibilidade e Programas Urbanos do Ministério das Cidades (SNAPU/ MCidades); d) CEMADEN/MCTI; e) Agência Nacional de Águas, vinculada ao Ministério do Meio Ambiente (ANA/MMA). O roteiro de entrevista semiestruturada foi montado após a inspeção dos dados e análise de documentos, visando esclarecer aspectos imprecisos da análise até então realizada, e os atores sociais representativos das instituições foram escolhidas pelo seu envolvimento direto nas políticas relacionadas à prevenção de desastres. Com base na combinação da análise documental com o conteúdo das entrevistas, configurando uma triangulação de dados (Jick, 1979), buscou-se avaliar o nível de implementação do QAH na prevenção de desastres de origem hídrica no Brasil, sob o ponto de vista da valorização da prevenção de desastres nas políticas públicas, com base no trabalho de Manyena (2013). Esse autor organizou um conjunto de indicadores para Zimbábue baseados no QAH para avaliação do nível de qualidade das políticas relacionadas à gestão de desastres no contexto da prevenção do cólera naquele país, cuja estrutura pode ser utilizada em outros países ou para temas como os desastres de origem hídrica. Esses indicadores foram agrupados em cinco grandes áreas estratégicas:

a) governança (QAH-AE1)-organizacional, le- 
gal e política, com quatro indicadores;

b) identificação dos riscos (QAH-AE2)-avaliação, monitoramento e alerta precoce, com quatro indicadores;

c) gestão do conhecimento (QAH-AE3)-utilização do conhecimento, da inovação e da educação para criar uma cultura de segurança e resiliência em todos os níveis, com quatro indicadores;

d) redução de risco (QAH-AE4)-diminuição dos fatores de risco subjacentes, ou seja, das vulnerabilidades, com seis indicadores;

e) fortalecimento da capacidade de resposta (QAH-AE5)-melhoria da preparação para situação de desastres, assegurando uma resposta eficaz em todos os níveis de preparação, com quatro indicadores.

Essa estrutura de agrupamento de indicadores corresponde às áreas estratégicas do QAH, e além de Manyena (2013), também foi tomado como base para avaliar o esforço de contribuir na redução de desastre por meio da educação por Zhou et al. (2014). Manyena (2013) usou esses indicadores para avaliação por atores-chave por meio de entrevista, em que o entrevistado avaliava, segundo sua percepção, a eficiência das políticas públicas numa escala de ordenamento contendo três classes (baixa, média e alta). No entanto, neste nosso estudo, as entrevistas não foram utilizadas para avaliação do sistema. A avaliação do sistema, usando os mesmos indicadores de Manyena (2013), foi feita pelos próprios pesquisadores a partir da análise documental, e, portanto, de forma qualitativa. As entrevistas realizadas vieram apenas a esclarecer pontos da avaliação em que os dados não eram claros. De qualquer forma, adota-se a estrutura de agrupamento e de indicadores de Manyena (2013) aplicado à avaliação do sistema de prevenção e mitigação de desastres de origem hídrica no Brasil.

\section{Resultados e discussão}

Os resultados da avaliação qualitativa realizada decorrem do cruzamento entre indicadores desenvolvidos por Manyena (2013) detalhando cada uma das cinco áreas estratégicas para aumentar a resiliências das cidades aos riscos de desastres, com foco naqueles resultantes de inundações, enxurradas e alagamentos, descritos a seguir.

\section{1. Área Estratégica 1 (QAH-AE1) -} Governança: tornar a redução de risco de desastres uma prioridade nacional e local

\subsubsection{QAH-AE1.1 Arcabouço institucional e legal nacional para redução de risco de desastre existe, com responsabilidade e capacidade descentralizada em (todos os níveis)}

O Brasil iniciou o desenvolvimento do seu Sistema Nacional de Defesa Civil (SINDEC) a partir de 1942. O Serviço da Defesa Civil passou por diversos Ministérios, mas a criação de um sistema nacional de defesa civil deu-se por meio do Decreto ${ }^{\circ}{ }^{\circ}$ 97.274, de 16/12/1988. A partir da Constituição de 1988, compete à União planejar e promover a defesa permanente contra as calamidades públicas, especialmente as secas e as inundações (artigo 21, inciso XVIII). O Decreto n. ${ }^{\circ} 895 / 1993$, exigiu que o então SINDEC se articulasse nos três níveis de governo e 
passasse a contar com procedimentos padronizados na fase de resposta, relacionados à coordenação do cenário e atendimento a requerimentos burocráticos de avaliação de danos (Valencio, 2010).

A partir de 2012, o Brasil deu início à alteração de seu sistema normativo relativo à gestão de riscos e desastres naturais, seja em função dos eventos ocorridos em anos anteriores (inundações em Santa Catarina em 2008, inundações em Alagoas e Pernambuco em 2010, e deslizamentos na Região Serrana do Rio de Janeiro em 2011), seja em função da adesão ao QAH em 2011: a instituição da Política Nacional de Proteção e Defesa Civil (PNPDEC) que dispôs sobre o Sistema Nacional de Proteção e Defesa Civil (SINPDEC) e sobre o Conselho Nacional de Proteção e Defesa Civil (CONPDEC) (Lei n. ${ }^{\circ}$ 12.608, de 2012 e Instrução Normativa n. ${ }^{\circ}$ 1, de 24 de agosto de 2012, do Ministério da Integração Nacional).

O indicador proposto por Manyena (2013) para essa ação QAH-AE1.1 é que haja disponibilidade de marcos legais para redução de risco de desastres. Verifica-se que há normativos institucionais e legislativas nacionais, sendo positiva a resposta para esse indicador, ou seja, o indicador foi atendido.

\subsubsection{QAH-AE1.2 Recursos dedicados e adequados estão disponíveis para implementar planos para redução de risco de desastres em todos os níveis administrativos}

O Plano Plurianual 2012-2015 incluiu o Programa 2040 - Gestão de Riscos e Respostas a Desastres, tendo alocado recursos da ordem de 18,8 bilhões de reais para o período. A inclusão do Programa 2040 atestou o compromisso assumido pelo governo com a gestão de riscos e respostas a desastres no país.

A Lei n. ${ }^{\circ} 12.340$ de 2012, estabeleceu duas formas de transferência de recursos financeiros para a execução de ações de prevenção em áreas de risco de desastres e de resposta e de recuperação em áreas atingidas por desastres aos órgãos e entidades dos Estados, Distrito Federal e Municípios: i) depósito em conta específica mantida pelo ente beneficiário em instituição financeira oficial federal, e ii) do Fundo Nacional para Calamidades Públicas, Proteção e Defesa Civil (Funcap) a fundos constituídos pelos Estados, Distrito Federal e Municípios. Os depósitos em contas específicas objetivam atender às ações de resposta, enquanto que os recursos transferidos por meio do Funcap têm por finalidade custear ações de prevenção em áreas de riscos de desastres e de recuperação em áreas atingidas por desastres, mediante reconhecimento da situação de emergência ou de calamidade pública do ente federado.

As atividades voltadas para as respostas aos desastres tornaram-se prioritárias e mais ágeis a partir da instituição do Cartão de Pagamento da Defesa Civil (CPDC) por meio do Decreto n. ${ }^{0} 7.257$ de 2010, que permite aos Estados, Distrito Federal ou Municípios terem a situação de emergência ou o estado de calamidade pública reconhecida e fazer jus ao recebimento dos recursos transferidos pela União para conta específica do beneficiário em instituição financeira oficial federal. A questão orçamentária e financeira é considerada fundamental num quadro de ação para gestão de risco de desastre e sua prevenção e mitigação segundo Ahmed (2013).

Considerando que o indicador proposto para a ação QAH-AE1.2 é que haja disponibilidade de recursos orçamentários para o planejamento e implementação de ações com vistas à redução de risco 
de desastres, verifica-se que existem as estruturas orçamentárias necessárias para a disponibilização dos recursos financeiros. Diante do exposto, tem-se por atendido o indicador ora avaliado.

\subsubsection{QAH-AE1.3 Participação da comunidade e descentralização por meio de delegação de autoridade e recursos para niveis locais}

Do conjunto normativo analisado pode-se inferir que a população ainda é tratada, basicamente, como vítima, portanto, como agente passivo que necessita de socorro e de assistência. Nesses instrumentos legais, a população é vista como passiva aos desastres, sem protagonismo, mesmo quando se trata de prevenção, posto que não é estimulada a participar das citadas ações e comparece, apenas, como agente receptor de informações às vésperas do desastre ou no momento em que a tragédia acontece. O papel reservado à população é o de participação em exercícios simulados, quando oferecidos pelo Poder Público, ou por meio de organizações comunitárias de caráter voluntário com atuação nas ações de proteção e defesa civil. Essa temática da participação merece ser destacada, pois Hakaloba et al. (2016) informam tratar-se de orientação cada vez mais aceita no setor, que passou por uma mudança da abordagem top-down para uma nova abordagem centrada nas pessoas, que inclui, entre outros, a participação dos atores sociais e a mudança ou transferência de responsabilidades das autoridades para o público. Ou seja, é preciso que a participação seja de fato efetiva, diferentemente do que se constatou na presente pesquisa, para que a participação não seja apenas passiva e limitada a situações previa- mente definida pela autoridade, configurando uma distorção do princípio já caracterizada como mito da participação em Santos \& Saito (2006).

A PNPDEC estabeleceu como uma de suas diretrizes a participação da sociedade civil, mas a limitou em aproximadamente $16 \%$ dos membros do CONPDEC, ou a $23 \%$ de acordo com o artigo 4. ${ }^{\circ}$ da Portaria n. ${ }^{\circ} 139$, de 18 de abril de 2013, que aprovou o Regimento Interno do CONPDEC e incluiu dois representantes das comunidades atingidas por desastres e dois especialistas de notório saber, ao arrepio da lei. O mito da participação social (Santos \& Saito, 2006) está presente na PNPDEC, visto que a participação é apresentada como uma dádiva, uma concessão, em um ambiente controlado pelas instâncias governamentais que detêm a ampla maioria do CONPDEC.

Ao que parece não houve a participação popular preconizada pelo QAH, nem tampouco resta estabelecida a rede de organizações voluntárias, no que pese o arcabouço normativo construído. Como a participação da comunidade e a descentralização por meio de delegação de autoridade e recursos para níveis locais, até o momento, não foi efetivada, considera-se que esse indicador não foi atendido.

\subsubsection{QAH-AE1.4 Plataforma nacional} multisetorial para funções de redução de risco de desastres

A PNPDEC estabelecida a partir de 2012 está parcialmente traduzida no Programa 2040 de Gestão e Prevenção de Riscos e Respostas a Desastres Naturais do PPA 2012-2015, o que reflete a adoção de uma política pública que busca contemplar diferentes setores governamentais - planejamento, 
serviço geológico, mapeamento do uso do solo urbano, disponibilização de tecnologia via centros de monitoramento e alerta, atuação direta da Defesa Civil em todos os níveis federativos -, e expressa a intenção em alcançar uma ação coordenada e multidisciplinar no âmbitos da prevenção, do mapeamento, do monitoramento e do alerta, e das respostas aos desastres naturais.

A multisetorialidade preconizada pelo QAH e usada como indicador por Manyena (2013) está estabelecida. Todavia, registre-se que apenas três dos seis atores que participaram da elaboração do PPA 2012-2015 também participam da CONPDEC, sendo que este conta com 12 atores. A leitura das Atas disponíveis do CONPDEC evidencia a busca da multisetorialidade pelos atores e o desejo de alcançar a sinergia necessária para o funcionamento do SINPDEC. Entretanto, considera-se que a sincronia entre os diversos atores ainda está em construção e verifica-se a necessidade de maior interação entre o órgão consultivo (CONPDEC) e a instância executiva (PPA 2012-2015) em favor da eficiência e eficácia do SINPDEC. Diante do exposto, tem-se por parcialmente atendido esse indicador.

\section{2. Área Estratégica 2 (QAH-AE2) -} identificação dos riscos: melhorando o conhecimento do risco

\subsubsection{QAH-AE2.1 Avaliação de risco local e nacional baseado em dados de perigo e informações de vulnerabilidade estão disponíveis e incluem a avaliação de risco para setores chaves.}

A Companhia de Pesquisa de Recursos Minerais do Ministério das Minas e Energia (CPRM/ MME) mapeia os 821 municípios críticos do Plano Nacional de Gestão de Riscos e Resposta a Desastres Naturais, selecionados pelo conjunto dos órgãos envolvidos na concepção do Programa 2040. A CPRM/MME analisa também o risco em uma região urbana em escala de 1:2.000 a 1:1.000, produzindo um mapa com dados vetoriais e base de dados da região, além de fotos. Todo conhecimento produzido é transferido para o município, para o CEMADEN/MCTI, CENAD/MI, MCidades e demais órgãos e instituições integrantes do Plano Nacional de Gestão de Riscos e Resposta a Desastres Naturais (Sampaio et al., 2013). Além disso, o MCidades apoia a elaboração de cartas geotécnicas de aptidão à urbanização em 106 municípios, de 12 Estados. As cartas geotécnicas são elaboradas com base em levantamentos geológicos e geotécnicos, na escala de 1:10.000, e na legislação que dispõe sobre o parcelamento do solo urbano e sobre defesa civil e visam ao planejamento urbano e planos diretores. Essas cartas são encaminhadas para CEMADEN/ MCTI e subsidiam os alertas, quando necessários, a serem emitidos pelo CENAD/MI. O conhecimento atualizado dos riscos por meio de um efetivo monitoramento é considerado fundamental por Dash $e t$ al. (2016). No entanto, para Manyena (2013), não basta que as informações sejam elaboradas pelas instituições nacionais, é necessário que estejam disponíveis e acessíveis, inclusive para que a população possa contribuir na tomada de decisão, condição ainda não alcançada pelo Brasil. Dessa forma, tem-se por parcialmente atendido esse indicador. 
3.2.2. QAH-AE2.2 Sistemas estão em condições para monitorar, armazenar e disseminar dados sobre perigos-chave e vulnerabilidades

A rede de observação do SINPDEC conta com radares meteorológicos, estações hidrológicas e meteorológicas, sensores de umidade do solo e de deslizamentos, sob o gerenciamento do CEMADEM/MCTI que, após processar os dados recebidos, os disponibiliza aos órgãos integrantes do sistema de proteção e defesa civil e à população em geral, por meio de mapas interativos. Os sistemas desenvolvidos até o momento, aparentemente, estão em condições de monitorar e armazenar informações. Todavia, o mesmo não se pode afirmar sobre os sistemas de previsão e alerta de inundação, que, em geral, não exigem grandes investimentos, mas precisam ser bem entendidos pela sociedade para serem efetivos, o que desafia a participação social.

Ainda que a academia tenha desenvolvido inúmeros sistemas de alerta precoce (Dantas, 2012; Gonçalves, 2009; Andrade, 2006), ao que parece, ainda não estão ao alcance da coletividade, ou seja, não foram revelados para uso comunitário ou, ainda, a comunicação entre os sistemas de alerta e a coletividade não foi devidamente estabelecida. Diante do todo exposto, conclui-se que o Brasil desenvolveu e desenvolve condições para monitoramento meteorológico, hidrológico e geológico das áreas de risco, para armazenar e disseminar (ainda que em nível institucional) dados sobre perigos-chave e vulnerabilidades, mas não para disseminar o alerta sobre perigos e vulnerabilidades, razão pela qual tem-se por parcialmente atendido o indicador em tela.

\subsubsection{QAH-AE2.3 Sistemas de alerta precoce estão colocados para os grandes perigos, com alcance às comunidades}

$\mathrm{O}$ aparato tecnológico colocado à disposição do CEMADEN/MCTI somado ao monitoramento realizado pela CPRM/MI e por outros órgãos do sistema possibilita, de acordo com os gestores, a emissão de alerta com prazo de duas a seis horas de antecedência.

Destaque-se que o CEMADEN/MCTI desenvolve o Projeto Pluviômetros nas Comunidades, que tem por objetivo permitir que a sociedade tenha acesso a dados pluviométricos em tempo real e se mobilize para mitigar os efeitos do desastre natural iminente. Esse projeto capacita a população local para instalar os pluviômetros semiautomáticos e a entender os resultados apresentados em um visor digital (ou display). O sistema permite o armazenamento de dados (ou datalogger), mas é possível a conexão de um dispositivo externo para a retirada dos dados armazenados, os quais poderão ser transmitidos manualmente por equipes das comunidades das áreas de risco para os órgãos de monitoramento de desastres naturais. O referido projeto teve início em 2012 e atualmente está instalado em 21 (vinte e um) Estados, contempla 14 (quatorze) capitais e 305 (trezentos e cinco municípios), vulneráveis à ocorrência. Esse projeto, até o momento, está disponível em $37 \%$ do total dos 821 (oitocentos e vinte e um) municípios inicialmente selecionados pelo Poder Executivo para serem monitorados e já apresenta um quantitativo absoluto superior aos 286 municípios prioritários para monitoramento pelo CEMADEN/MCTI. 
Por meio da Portaria Conjunta (ANA/MMA, CEMADEN/MCTI, CENAD/MI, CPRM/MME) n. ${ }^{\circ}$ 148, 18 de dezembro de 2013, instituiu-se protocolo para especificar, de modo exclusivo, a competência e as ações referentes à emissão de alertas para inundações. Essa Portaria atribui ao CENAD/MI a responsabilidade pela expedição do aviso à Defesa Civil dos Estados e Municípios. A Portaria visa disciplinar a relação entre as instituições governamentais e não contempla a participação da população. Ressalte-se que o alerta pode ocorrer por meio da presença do agente da Defesa Civil no local de risco e até por meio de telefone celular (serviço móvel pessoal), sendo que as empresas estão obrigadas por lei (art. 15-B, da Lei n. ${ }^{\circ}$ 12.340/2010) a transmitir gratuitamente informações de alerta à população sobre risco de desastre.

Conclui-se, portanto, que o indicador foi parcialmente atendido. $\mathrm{O}$ indicador seria plenamente atendido se o Projeto já contemplasse, pelo menos, todos os 821 municípios inicialmente selecionados para monitoramento, pois aí todas as comunidades de risco estariam contempladas.

\subsubsection{QAH-AE2.4 Avaliação de risco local e nacional leva em consideração riscos regionais e transfronteiriços, com uma visão para a cooperação regional para redução de risco.}

O desafio de monitorar 821 municípios com vistas a obter um mapeamento das áreas sujeitas a eventos hidrológicos, além de eventos geológicos, assim como o desenho institucional desenvolvido para alcançar tal objetivo, evidencia a cooperação interna, necessária e fundamental para atingir a meta estabelecida. O Programa 2040 explicita o compromisso e a cooperação entre os diversos níveis dos entes federativos.

Já o risco de desastres no âmbito regional (América do Sul), transfronteiriço ou no contexto internacional não foi identificado, seja na legislação, seja nos programas governamentais, evidentemente no que se refere a eventos de natureza hidrológica. Apesar da legislação brasileira sobre desastres não dispor sobre o contexto regional, consignou como princípio que rege as relações internacionais do Brasil a cooperação entre os povos para o progresso da humanidade e o desastre compromete significativamente o alcance desse estágio.

Para Manyena (2013), o indicador seria atendido se abordasse os riscos, especialmente transfronteiriços, tendo em vista que é preciso garantir a resiliência não apenas das comunidades que ocupam as fronteiras dos países, mas da região e, por consequência, do próprio país na hipótese de ocorrência de desastres. Diante do todo exposto, observa-se que o indicador em tela não foi atendido.

\subsection{Area Estratégica 3 (QAH-AE3) -} gestão do conhecimento: Utilização do conhecimento, inovação e educação para construir resiliência

\subsubsection{QAH-AE3.1. Informações relevantes} em desastres estão disponíveis e acessiveis em todos os níveis, para todos os atores sociais (por meio de redes e desenvolvimento de um sistema de compartilhamento de informação) 
O Brasil conta com a Lei n. ${ }^{\circ} 12.527$, de 2011, que garante e estabelece procedimentos para o acesso a informações de interesse particular do cidadão e, inclusive, de interesse coletivo ou geral. Nessa lei, também designada de Lei de Acesso à Informação (LAI), considera-se informação, dados, processados ou não, que podem ser utilizados para produção e transmissão de conhecimento, contidos em qualquer meio, suporte ou formato (Brasil, 2011), sendo dever do Estado assegurar o acesso à informação.

No que pese o aparato legislativo disponível, observa-se que as informações produzidas sobre desastres ainda não estão acessíveis, tampouco foram transformadas em conhecimento à população. No sítio do CENAD/MI existem algumas dicas de como proceder no caso de inundações e outros eventos. Já a informação disponibilizada pelo CEMADEN/ MCTI é de natureza científica e, portanto, endereçada ao público especializado.

Verifica-se que, se de um lado, foi estabelecido um protocolo de comunicação e alerta entre organismos governamentais, ainda que exclusivamente para os eventos de inundação (Portaria Conjunta ANA/MMA, CEMADEN/MCTI, CENAD/MI, CPRM/MME n. ${ }^{\circ}$ 148, de 18 de dezembro de 2013), por outro, a disseminação da informação para a população permanece sem regramento e afeta a população em todas as fases do desastre, pois a informação estimula a prevenção, assim como a logística a ser adotada durante e após o desastre.

Verifica-se, por consequência, que os sistemas de alerta ainda não foram implantados ou desenhados de forma acessível à população, podendo-se concluir que o indicador dado por Manyena (2013), cuja disponibilidade de informações é relevante para todos os atores sociais, de modo a permitir a resiliência às inundações, não foi atendido.
3.3.2. QAH-AE3.2 Currículo escolar, material educacional e treinamento relevante incluem redução de risco e recuperam conceitos e práticas

As ações de prevenção, mitigação, preparação e resiliência ainda não integram os currículos escolares, bem como os materiais educacionais são esparsos e possivelmente não atingem a população vulnerável aos eventos hidrológicos, ainda que o desenvolvimento de consciência nacional sobre os desastres se constitua em um dos objetivos da PNPDEC.

É importante lembrar que os conhecimentos necessários para mitigar os riscos de inundações serão adequados se beneficiarem a coletividade afetada e se estiver fundamentada em princípios científicos sedimentados. O papel mediador do conhecimento científico ocupa um lugar importante, sobretudo para viabilizar processos participativos e de justiça social e ambiental (Santos et al., 2005). Esse fato é ainda mais importante ao se considerar que o acesso ao conhecimento é desigual na sociedade, da mesma forma como a vulnerabilidade a desastres. De acordo com Beck (2006), o risco catastrófico segue os pobres. Para esse autor, os riscos globais teriam duas faces: a probabilidade de possíveis catástrofes e a vulnerabilidade social a essas catástrofes.

A produção e elaboração de material didático-pedagógico relacionado ao desenvolvimento da cultura de prevenção de desastres é de competência da União por meio da comunidade docente respectiva (Brasil, 2012), entretanto, não foi possível detectar iniciativas nesse sentido relacionadas a eventos de inundação, enxurradas e alagamentos.

Conclui-se que não se verificou a inclusão da 
redução de risco de desastres e seus conceitos nos currículos escolares, em material educacional e de treinamento, de maneira que se tem por não foi atendido o indicador sugerido por Manyena (2013).

\subsubsection{QAH-AE3.3 Métodos de pesquisa e ferramentas para avaliação multirrisco e análise de custo-benefício são desenvolvidas e fortalecidos}

Apesar de as ações estarem ainda restritas a pequenos grupos de instituições ocasionais, observa-se a consolidação de instituições e ações mais perenes, como a Fundação Oswaldo Cruz, que desenvolveu em sua estrutura o Centro de Estudos e Pesquisas em Emergências e Desastres em Saúde (Cepedes/ Fiocruz) e recentemente lançou o sítio denominado Centro de Conhecimento em Saúde Pública e Desastres, e o Centro de Estudos e Pesquisas em Desastres (Ceped) da Universidade Federal de Santa Catarina.

O próprio Banco Mundial promoveu avaliações de perdas e danos resultantes dos desastres hidrológicos que afetaram os estados de Santa Catarina, Alagoas e Pernambuco e a Região Serrana do Rio de Janeiro, por meio da metodologia DaLe (Toro, 2014). Tais avaliações são as únicas disponíveis no Brasil e apontam um caminho ainda a ser percorrido pelo país.

Apesar dos esforços concretos no sentido de se alcançar uma ação articulada e uma abordagem sistêmica das ações de prevenção, mitigação, preparação, resposta, recuperação e, inclusive, no âmbito da pesquisa científica, o indicador seria atendido para Manyena (2013) se houvesse evidências de que as instituições envolvidas, especialmente com a pesquisa interdisciplinar, estivessem em conexão, por meio, por exemplo, de eventos que avaliassem as perdas de vidas, o deslocamento das populações, os danos ao ambiente, às infraestruturas pública e privada, o comprometimento da saúde das populações atingidas, e o comprometimento do desenvolvimento econômico. O que prevalece ainda são iniciativas mais pontuais e isoladas como em Machado et al. (2005), os quais propuseram uma metodologia que combina os métodos a posteriori e a priori para avaliar o impacto das enchentes sobre o município de Itajubá-MG. Finalmente, cabe destacar que os sistemas de avaliação de desastres não medem os danos subsequentes e, muitas vezes imperceptíveis, pela sociedade e pelo estado, ou seja, ainda há carência de uma visão sistêmica que se preocupe com os aspectos menos visíveis e menos imediatos (Saito, 2013). A conexão entre as diversas áreas de conhecimento para a gestão dos desastres parece que ainda não foi alcançada, dessa forma, considera-se não atendido o indicador em questão.

\subsubsection{QAH-AE3.4 Estratégia de consciência} pública existe em todo o país para estimular uma cultura de resiliência a desastres, com alcance para comunidades urbanas e rurais

Não se verificou a existência de qualquer estratégia nacional de comunicação com vistas a estimular a população para o desenvolvimento de uma cultura de prevenção, mitigação, preparação, resposta, recuperação capaz de tornar resiliente o meio urbano aos desastres naturais de origem hidrológica, ainda que o desenvolvimento da consciência nacional e a orientação das comunidades para a prevenção e respostas em situação de desastres se constituam em objetivos políticos (Brasil, 2012). 
Se não há informação disponível sobre o meio em que se habita será muito difícil conhecer os riscos potenciais do local, visto que o conhecimento é a melhor maneira de proteção (Kobiyama et al., 2006) e, porque não dizer, de prevenção. Considera-se que as informações não estão disponíveis e que, se de um lado pode até existir consciência da população sobre os riscos que a cercam, por outro, parece não haver clareza sobre como mitigá-los, ou sobre como tornar-se resiliente a eles.

Diante do exposto, outra não pode ser a conclusão senão que o indicador proposto por Manyena (2013) não foi atendido.

\section{4. Área Estratégica 4 (QAH-AE4)-} redução de risco: diminuição dos fatores de risco subjacentes

\subsubsection{QAH-AE4.1 Redução do risco de} desastres é um objetivo integral das políticas e dos planos relacionados ao meio-ambiente, incluindo uso da terra, gestão de recursos naturais e adaptação a mudanças climáticas

O Brasil tem sido profícuo na produção de políticas públicas. Além das políticas nacionais sobre diferentes temas, a PNPDEC, quando foi criada, the foi atribuído o dever de integrar-se às políticas de ordenamento territorial, desenvolvimento urbano, meio ambiente, mudanças climáticas, além das políticas de saúde, gestão de recursos hídricos, geologia, infraestrutura, educação, ciência e tecnologia e às demais políticas setoriais, tendo em vista a promoção do desenvolvimento sustentável por determinação legal (parágrafo único, art. $3^{\circ} \mathrm{da}$
Lei n. ${ }^{\circ}$ 12.608, de 2012).

$\mathrm{Se}$, de um lado, os documentos legais preconizam integração das políticas, por outro, os documentos pesquisados indicam que essa conexão entre os diversos setores governamentais ainda não atingiu a eficácia pretendida. A integração entre as políticas públicas, por enquanto, está restrita a seis ministérios (MPOG, MI, MMA MCidades, MCTI e MME), contempla partes dessas estruturas, quando deveria interagir (por determinação legal) com os Ministérios da Saúde e da Educação para produzir mudanças estruturais, universais e capazes de impactar o comportamento dos indivíduos tal que atendam aos interesses gerais da sociedade.

O desafio de integrar a PNPDEC está posto, cristalizado em lei, mas sua eficácia é parcial, haja vista que, apesar de ter integrado diversas áreas por meio do Programa 2040, não absorveu a área de saúde - fundamental para a fase de prevenção e de recuperação -, assim como a área de educação, essencial para a fase de prevenção.

Esse aspecto é merecedor de um comentário adicional, tendo em vista que Isaranuwatchai et al. (2016) constataram que, no período entre um e dois anos após o tsunami de 2004 na Tailândia, o uso de todos os serviços de saúde aumentou de modo geral tanto para os grupos afetados quanto para os não afetados diretamente pelo tsunami. Os autores enfatizam o fato de que, muitas vezes, organizações e governos não antecipam o efeito duradouro de um desastre natural e retiram prematuramente os serviços de apoio às pessoas afetadas, ou diminuem os investimentos em setores de atendimento às vítimas, como o de saúde, após um curto prazo pós-desastre.

Adicionalmente, cabe lembrar que o impacto das alterações climáticas sobre os padrões meteorológicos, por sua vez, favorece o risco de 
inundações (Jha et al., 2012; Marengo, 2008), e apesar de reconhecido no âmbito legislativo, não foi plenamente incorporado à práxis governamental, nem se percebe a transversalidade preconizada nos documentos de planejamento do Estado.

Assim, diante de todo os aspectos analisados, considera-se não atendido o indicador proposto por Manyena (2013).

\subsubsection{QAH-AE4.2 Políticas e planos de desenvolvimento social são implementadas para reduzir a vulnerabilidade das populações que mais se encontram em risco}

As políticas estão postas no plano nacional e divididas em medidas de natureza estrutural e não estrutural. Elas têm se tornado efetivas por meio do Programa 2040.

Ressalta-se que os recursos destinados no PPA às medidas estruturais, definidas pelas políticas públicas, são da ordem de R $\$ 9$ bilhões para apoio à execução de intervenções de drenagem urbana sustentável nos municípios mais suscetíveis a desastres associados a enchentes e inundações recorrentes em áreas urbanas, além de intervenções emergenciais.

Já para as medidas não estruturais foi destinada a importância de $\mathrm{R} \$ 9,8$ bilhões no orçamento da União. Essas medidas estão representadas por mapeamentos, modelagens geodinâmicas e hidrológicas, sistemas de alerta, capacitação, cooperação para estruturação de órgãos de defesa civil regionais e municipais, modernização e estruturação dos órgãos que compõem o SINDEC, além de assistência às populações vitimadas e reabilitação dos cenários de desastres.

É possível observar, também, que existem po- líticas voltadas para redução de desastres que, apesar de não estarem descritas ou incorporadas no Programa 2040, estão a ser implementadas para redução da vulnerabilidade das populações que mais se encontram em risco, tais como programas habitacionais que objetivam a regularização fundiária e de prevenção de riscos. O primeiro visa tornar o solo urbano ocupado legal e sustentável ambientalmente e o segundo evitar os desastres de origem hidrológica.

Todavia, verifica-se que há um vazio de políticas relacionadas à utilização do conhecimento, inovação e educação para construir resiliência. Dessa forma, considera-se que o indicador proposto por Manyena (2013) está parcialmente atendido.

\subsubsection{QAH-AE4.3 Politicas e planos econômicos e para setores produtivos são implementadas para reduzir a} vulnerabilidade das atividades econômicas

Os setores produtivos ainda não estão envolvidos com a PNPDEC nem há previsão formal para sua inclusão. As perdas e danos decorrentes de desastres provocados por inundações, enxurradas e alagamentos são suportadas pela iniciativa privada, ao que tudo indica, sem que ela perceba a necessidade de proteção financeira contra os mencionados eventos hidrológicos, sem que compreenda a possibilidade de prevenção e mitigação desses eventos mas, principalmente, sem perceber sua capacidade de tornar-se resiliente ante a eles.

O Relatório do Banco Mundial (Toro, 2014) que avalia as opções de proteção do Brasil diante de eventos críticos, tais como inundações, informa que tais opções estão restritas ao seguro agrícola e 
ao seguro de propriedade.

O seguro agrícola vem crescendo desde 2003, notadamente em virtude do Programa de Subvenção do Governo e da Lei Complementar n. ${ }^{\circ}$ 137, de 2010, que autorizou a participação da União em fundo destinado à cobertura dos riscos do seguro rural. No caso dos seguros agrícolas, a exposição a eventos hidrológicos e climatológicos e a possibilidade de remediar os riscos dessas exposições deve ter contribuído para que o setor buscasse se proteger das vulnerabilidades.

No que se refere aos seguros de propriedade que contemplam a garantia de perdas, danos ou responsabilidades sobre objetos ou pessoas, eles foram ampliados para incluir coberturas contra eventos naturais, mas as seguradoras apresentam como gargalos ao crescimento do setor a falta de acesso a informações relevantes, tais como perdas históricas resultantes de desastres, mapas de risco atualizados, modelagem de perigos e ferramentas de monitoramento climático, dados esses que dificultam a precificação adequada do seguro (Toro, 2014).

A ausência de representantes dos setores agrícola, industrial e comercial no CONDEC talvez contribua com a reduzida percepção do setor sobre o risco representado pelos eventos críticos e para as alternativas de proteção oferecidas tanto pelo governo quanto pelas seguradoras.

A oportunidade apresentada para a redução das vulnerabilidades por meio de seguros para o setor privado, inclusive com subvenção pública para alguns segmentos econômicos específicos, parece ampla, mas não é percebida pelos interessados. Assim, considera-se não atendido o indicador proposto por Manyena (2013).

\subsubsection{QAH-AE4.4 Planejamento e gestão} de assentamentos humanos incorporam elementos de redução de risco de desastres, incluindo aplicação de códigos de edificações

O Estatuto da Cidade (Lei n. ${ }^{\circ} 10.257$, de 10 de julho de 2001), também designada de Lei do Meio Ambiente Artificial (Santin \& Marangon, 2008; Fiorillo, 2002) foi alterado simultaneamente à norma que dispõe sobre PNPDEC (Brasil, 2012) para estabelecer que a política urbana tem por objetivo ordenar o pleno desenvolvimento das funções sociais da cidade e, dessa forma, deve evitar exposição da população a riscos de desastres. A alteração promovida no Estatuto da Cidade obriga os municípios com áreas suscetíveis a eventos hidrológicos a elaborar seu Plano Diretor, devendo os mencionados Planos identificar e mapear as áreas de risco, além de planejar ações preventivas de risco.

Sobre a ordem urbanística incide o planejamento, que já não depende da vontade de um governante, posto que exigido por lei e que deve ser capaz de alterar a realidade para atender aos objetivos previamente estabelecidos (Silva, 2000).

Em vista do exposto, pode-se concluir que o indicador proposto por Manyena (2013) foi atendido. Cabe salientar ainda que, para que esse quesito possa ganhar maior efetividade ao longo do tempo, é necessário investir na formação profissional de recursos humanos na área de edificações e planejamento urbano, para que incorporem a perspectiva de prevenção de desastres. A indústria da construção e as profissões que nela atuam têm um papel importante a desempenhar na contribuição para a resiliência melhorada da sociedade, conforme alerta Haigh \& Amaratunga (2010). 
3.4.5. QAH-AE4.5 Medidas de redução do risco de desastres estão integradas à recuperação pós-desastres e processos de reabilitação

Observa-se que as ações de redução de risco estão conectadas com as medidas pós-desastres, especialmente por meio do CPDC, tendo em vista que a União é obrigada por lei a efetuar transferências aos estados, ao Distrito Federal e aos municípios para execução de ações de prevenção em áreas de riscos de desastres e de resposta e de recuperação em áreas atingidas (Brasil, 2010).

Destaque-se que a transferência de recursos no momento pós-desastre pode ocorrer, independentemente do prévio reconhecimento federal, desde que devidamente motivado pelo ente a ser beneficiado.

O compromisso com a assistência está consignado nos instrumentos de planejamento, nas transferências obrigatórias para ações de socorro, assistência às vítimas, restabelecimento de serviços essenciais e reconstrução por meio do CPDC. Até outubro de 2014, o total de 2.089 municípios aderiram ao Cartão que se tornou operacional desde novembro de 2011, conforme informação obtida no Portal da Transparência da Controladoria-Geral da União.

Diante do exposto, conclui-se que o indicador proposto por Manyena (2013) foi atendido.

\subsubsection{QAH-AE4.6 Procedimentos estão} prontos para avaliar o impacto dos riscos de desastres para os grandes projetos de desenvolvimento, especialmente infraestrutura
A avaliação da infraestrutura pública e privada que poderá vir a ser afetada em caso de inundações, enxurradas e alagamentos ainda não integra as ações de mapeamento, tampouco de monitoramento do Poder Público. A pesquisa identificou apenas documentos do Banco Mundial que avaliam perdas e danos decorrentes de desastres de origem hidrológica (Toro, 2012a; 2012b; 2012c; 2012d).

$\mathrm{O}$ dimensionamento das perdas e danos provocado pelos desastres sobre as finanças da União, dos estados e dos municípios afetados ainda não possuem uma metodologia consolidada e adaptada aos eventos críticos incidentes sobre o país, especialmente no que tange às inundações. Diante desse quadro, torna-se necessário o desenvolvimento de uma estratégia de opções de proteção financeira contra desastres que considere os orçamentos de todos os entes federativos (Toro, 2014).

O indicador proposto por Manyena (2013), portanto, foi parcialmente atendido.

\section{5. Área Estratégica 5 (QAH5) -}

fortalecimento da capacidade de resposta: melhoria da preparação para situação de desastres, visando uma efetiva resposta em todos os niveis

\subsubsection{QAH-AE5.1 Política forte, capacidade} técnica e institucional e mecanismos para gestão de desastres com a perspectiva da redução de risco de desastres estão postos

Os avanços observados na alteração da base legal, a instituição do PNPDEC, a inclusão da Po- 
lítica no PPA 2012-2015 evidenciam uma decisão política de tratar do tema. A articulação construída entre os órgãos públicos é outro indicador da tentativa desses órgãos de superar possíveis zonas de sombreamento entre as respectivas atribuições e a busca pela adoção de medidas necessárias à redução dos riscos de desastres, com vistas a tornar as cidades resilientes a tais eventos.

Há uma tentativa clara de fortalecer instituições públicas e fazer cumprir (enforcement) leis. No caso dos desastres, sejam eles de origem hidrológica ou não, parece ter se configurado uma rara situação em que o interesse de todos (a soma dos indivíduos de uma comunidade) parece corresponder ao interesse público (o bem-estar geral da sociedade) (Bursztyn \& Bursztyn, 2012), configurando-se uma situação ideal para o exercício pleno da governança. Tem-se investido na capacitação técnica e científica por meio de instituições como CENAD/MI, CEMADEM/MCTI, CPRM/MME e ANA/MMA e, principalmente, na articulação das mencionadas instituições para o enfrentamento de inundações, conforme Portaria Conjunta n. ${ }^{\circ}$ 148, de 2013.

Observa-se, também, que a política pública para redução dos desastres está posta. Há o desenvolvimento de capacidades técnicas, assim como verifica-se um crescimento das ações voltadas para a gestão dos desastres, de maneira que se tem por atendido o indicador sugerido por Manyena (2013).

\subsubsection{QAH-AE5.2 Planos de prevenção de desastres e planos de contingência estão postos em todos os niveis administrativos, e treinamento instrucional regular e ensaio são adotados para testar e desenvolver programas de resposta a desastres}

Para a Portaria Conjunta n. ${ }^{\circ} 148$ de 2013, Plano de Contingência é o Planejamento realizado para controlar e minimizar os efeitos previsíveis de um desastre específico, contemplando: avaliação da ameaça de desastre; avaliação da vulnerabilidade do desastre; avaliação de risco; previsão de danos; avaliação dos meios disponíveis; estudo da variável tempo; estabelecimento de uma "hipótese de planejamento", após conclusão do estudo de situação; estabelecimento da necessidade de recursos externos, após comparação das necessidades com as possibilidades (recursos disponíveis); levantamento, comparação e definição da melhor linha de ação para a solução do problema; aperfeiçoamento e, em seguida, a implantação do programa de preparação para o enfrentamento do desastre; definição das missões das instituições e equipes de atuação e programação de "exercícios simulados, que servirão para testar o desempenho das equipes e aperfeiçoar o planejamento"

O conteúdo mínimo dos Planos de Contingência foi estabelecido em lei (Brasil, 2010) e contempla identificação e responsabilização de cada órgão na gestão do desastre, definição de sistemas de monitoramento e alerta, organização de exercícios simulados, organização de sistema de atendimento emergencial, ações de atendimento médico-hospitalar, cadastramento de equipes técnicas e de voluntários para atuar durante os desastres e identificação de centro de recebimentos e distribuição de doações.

Tanto no sítio do CEMADEN como no S2ID (Sistema Integrado de Informações sobre Desastres e o Observatório das Chuvas) inexiste indicação de qualquer sistematização capaz de indicar os Planos de Contingência elaborados pelos entes federativos.

Ainda, a legislação brasileira não contempla a modalidade Plano de Prevenção. Dessa forma, 
deve-se considerar como não atendido o indicador em análise.

\subsubsection{QAH-AE5.3 Reserva financeira e} mecanismos de contingência estão prontos para permitir resposta efetiva e recuperação quando requeridos

O principal instrumento de reserva financeira é o Funcap, criado em 1969 e preservado pela alteração legislativa promovida (Brasil, 2010), com a necessária atualização, mas não regulamentado até a presente data. O Funcap, de natureza contábil e financeira, será constituído, basicamente, por dotações na lei orçamentária anual da União e vinculado ao MI, todavia, o Distrito Federal, os estados e municípios deverão constituir seus próprios fundos para custear ações de prevenção em áreas de risco de desastres e de recuperação de áreas atingidas por desastres.

Atualmente, o que se observa é que a União transfere recursos aos demais entes federativos por meio do CPDC e que esses entes ainda não compuseram os respectivos fundos para emitir a resposta e efetivar ações de reconstrução quando da ocorrência de desastres. Diante do exposto, considera-se que a União tem efetuado a devida reserva de recursos em seu orçamento para permitir a resposta efetiva e a consequente recuperação, quando demandada, de maneira que se tem por atendido o indicador proposto por Manyena (2013).

\subsubsection{QAH-AE5.4 Procedimentos estão prontos para trocar informações relevantes durante desastres e lidar com revisões pós- eventos}

Há procedimentos integrados para a troca de informações durante os desastres, haja vista a institucionalização do Procedimento por meio da Portaria Conjunta n. ${ }^{\circ}$ 148, de 2013, especialmente para o caso de inundações. Por meio desse instrumento foi instituído o Plano de Chamadas, que visa estabelecer a comunicação de autoridades, de recursos humanos especializados em gestão de desastres, e do Comando Operacional Conjunto das Forças Armadas do Brasil, colocando-as de prontidão para agir no caso de concretizados desastres de grande magnitude.

Ademais, o Sistema Integrado de Informações sobre Desastres e o Observatório das Chuvas (S2id) possibilita a troca de informações entre a União e os municípios afetados por desastres, além de registrar e expedir o reconhecimento do desastre, transferir e fiscalizar os recursos transferidos quando da ocorrência de desastres.

No que se refere à avaliação pós-desastres, as lições aprendidas ainda não são rotineiras no âmbito governamental. Enquanto o monitoramento vai se estabelecendo como parte das políticas públicas, a avaliação das ações empreendidas ainda não foi incorporada. Diante do exposto e considerando que foram estabelecidos procedimentos para troca de informações relevantes durante desastres, mas que, no entanto, não há sistema de avaliação para lidar com revisões pós-eventos, considera-se parcialmente atendido o indicador em análise.

\subsection{Sintese dos indicadores}

A Tabela 1 apresenta uma avaliação final e sintética dos indicadores propostos por Manyena (2013) do QAH aplicados à realidade brasileira relacionado aos desastres de origem hídrica. 
TABELA 1 - Síntese da avaliação do nível de implementação do Quadro de Ação de Hyogo na prevenção de desastres no Brasil, sob o ponto de vista da valorização da prevenção de desastres nas políticas públicas

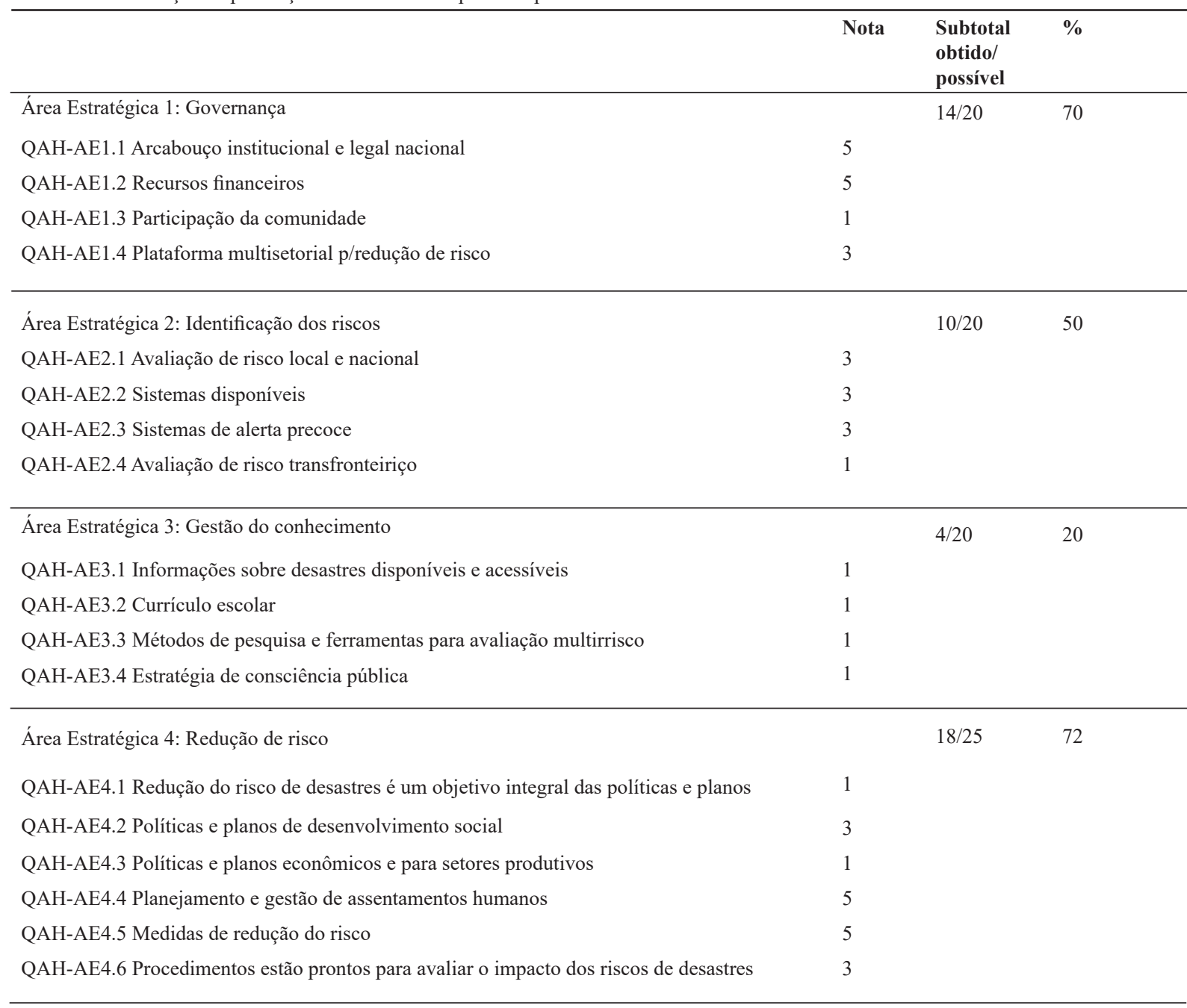

Área Estratégica 5: Fortalecimento da capacidade de resposta

QAH-AE5.1 Política forte, capacidade técnica e institucional

QAH-AE5.2 Planos de prevenção de desastres e planos de contingência 
Os dados obtidos revelam que o QAH ainda não é uma realidade no país. No entanto, esse resultado não significa que a responsabilidade por essa fragilidade na implementação do QAH seja apenas do governo federal, ou dos municípios. A responsabilidade deve ser partilhada também com o UNISDR.

A resposta ao desastre tem se estruturado mediante o uso do S2id, especialmente por esse sistema se constituir em uma ferramenta que registra e expede o reconhecimento do desastre, transfere e fiscaliza os recursos deslocados quando da ocorrência de desastres e visa disponibilizar banco de dados e registro de desastres, além de análise geoespacial e uma biblioteca virtual. O S2id é uma iniciativa pioneira, seja como instrumento processual, seja como banco de dados. Mas é importante ressaltar que a classificação e codificação dos desastres tem por base a antiga Codificação de Desastres, Ameaças e Riscos (CODAR) do MI, em detrimento da Classificação e Codificação Brasileira de Desastres (COBRADE), esta semelhante à do Banco de Dados Internacional de Desastres (EM-DAT), do Centro para Pesquisa sobre Epidemiologia de Desastres (CRED) da Organização Mundial de Saúde (OMS/ ONU) e a simbologia correspondente.

A biblioteca virtual e o banco de dados de registros de desastres e a análise geoespacial ainda carecem de aperfeiçoamento, seja quanto ao acesso como quanto ao conteúdo da informação prestada que, de tão simplificada, não favorece ao conhecimento de quem a busca nem a transparência pretendida.

Verifica-se, de um lado, que há condições técnicas e legais para divulgação da vulnerabilidade e do risco local tanto para a população quanto para os setores-chave. Por outro, é preciso incorporar solu- ções locais exitosas, que ampliem a base geodinâmica e observem as regionalidades da geodiversidade brasileira (Diniz, 2012). Recorda-se que o objetivo da produção de informação e desenvolvimento de sistemas é auxiliar planejadores a minimizar vulnerabilidades, mas, ao mesmo tempo, é preciso regular e fiscalizar os padrões de construções, conservar espaços coletivos, melhorar a salubridade urbana e o transporte (Bhattarai \& Conway, 2010).

\section{Conclusões}

A avaliação do nível de implementação do Quadro de Ação de Hyogo na prevenção de desastres no Brasil, sob o ponto de vista da valorização da prevenção de desastres nas políticas públicas, mostrou apenas uma performance mediana em termos da implementação de ações governamentais voltadas à prevenção de desastres no país.

$\mathrm{O}$ esforço normativo mostrou-se sistêmico, de modo contrário à aleatoriedade com que se dá a internalização dos atos internacionais. O desenvolvimento do Programa 2040 se dá de forma não protocolar, mas de forma integrada e articulada entre os diversos entes públicos sem que haja uma instância formal para tanto - fórum de discussão, dias de trabalho, seminários etc. Todavia, há regularidade no acompanhamento do Programa 2040 e cada ente tem clareza sobre seu papel para a gestão do risco e sobre as respostas a serem dadas quando os desastres naturais ocorrem.

O alto nível de articulação entre os órgãos federais foi destacado por todos os agentes públicos, especialmente entre os quatro eixos contemplados no PPA: prevenção, mapeamento, monitoramento e alerta e resposta. No entanto, apesar dessa percepção pelos atores sociais entrevistados, os documentos 
pesquisados indicam que a articulação intragovernamental ainda não atingiu o nível desejado posto que a integração entre as políticas públicas, por enquanto, está restrita a seis ministérios (MPOG, MI, MMA MCidades, MCTI e MME). A prevenção tem sido trabalhada tanto no âmbito da SNPDEC do MI como nas metas estabelecidas para a política urbana do MCidades. O mapeamento tem sido efetivado pela CPRM/MME em colaboração com informações oriundas do MMA e do CEMADEN/ MCTI, sendo que este último é responsável pelo monitoramento e pelo alerta à Defesa Civil. Mas ainda se faz necessária a integração com os Ministérios da Saúde e da Educação para ampliar a capacidade preventiva e responsiva no que se refere aos desastres.

Em que pese os esforços no campo normativo, orçamentário, e de mapeamento de riscos, a análise detectou destacadamente a fragilidade no desenvolvimento de uma cultura nacional de prevenção de desastres. Nesse aspecto, além de processos formativos, há carências no aspecto informativo também. Dentre eles, destaca-se a necessidade de ampla divulgação das ideias contidas no documento “Quadro de Ação de Hyogo 2005-2015: construindo a resiliência das nações e comunidades a desastres", bem como das diretrizes da Estratégia Internacional para a Redução de Desastres - EIRD (International Strategy for Disaster Reduction - ISDR).

\section{Agradecimentos}

Ao $\mathrm{CNPq}$, pelo apoio concedido na forma de bolsa de produtividade em pesquisa do segundo autor. Ao CAPA, pela revisão textual deste artigo.

\section{Referências}

Ahmed, Z. Disaster risks and disaster management policies and practices in Pakistan: A critical analysis of Disaster Management Act 2010 of Pakistan. International Journal of Disaster Risk Reduction, 4, 15-20, 2013.

Andrade, J. P. M. Previsão hidrometeorológica visando sistema de alerta antecipado de cheias em bacias urbanas. São Carlos, Dissertação (Mestrado em Hidráulica e Saneamento) - USP, 2006. Disponível em: $<$ http://www.teses. usp.br/teses/disponiveis/18/18138/tde-21112006-132907/ pt-br.php>

Beck, U. Living in the world risk society. Economy and Society, 35(3), 329-345, 2006.

Bhattarai, K.; Conway, D. Urban Vulnerabilities in the Kathmandu Valley, Nepal: Visualizations of Human/Hazard Interactions. Journal of Geographic Information System, 2(2), 63-84, 2010.

Brasil. Lei n. ${ }^{\circ} 12.340$, de 1 de dezembro de 2010. Dispõe sobre as transferências de recursos da União aos órgãos e entidades dos Estados, Distrito Federal e Municípios para a execução de ações de prevenção em áreas de risco de desastres e de resposta e de recuperação em áreas atingidas por desastres e sobre o Fundo Nacional para Calamidades Públicas, Proteção e Defesa Civil; e dá outras providências. Diário Oficial da União, n. 230, 6 de dez. de 2010, Seção 1, p. 1-2.

Brasil. Lei n. ${ }^{\circ}$ 12.527, de 18 de novembro de 2011. Regula o acesso a informações previsto no inciso XXXIII do art. 50, no inciso II do $\S 3^{\circ}$ do art. 37 e no $\S 2^{\circ}$ do art. 216 da Constituição Federal; e dá outras providências. Diário Oficial da União, edição extra, 11 de ago. de 2011, Seção 1, p. 1-4.

Brasil. Lei n. ${ }^{\circ}$ 12.608, de 10 de abril de 2012. Institui a Política Nacional de Proteção e Defesa Civil-PNPDEC; dispõe sobre o Sistema Nacional de Proteção e Defesa Civil-SINPDEC e o Conselho Nacional de Proteção e Defesa Civil-CONPDEC; autoriza a criação de sistema de informações e monitoramento de desastres; e dá outras providências. Diário Oficial da União, n. 70, 11 de abr. de 2012c, Seção 1, p. 1-4. 
Brasil. Ministério da Integração Nacional. Secretaria Nacional de Defesa Civil. Centro Nacional de Gerenciamento de Riscos e Desastres. Anuário brasileiro de desastres naturais: 2012. Brasília: CENAD, 2013.

Bursztyn, M. A.; Bursztyn M. Fundamentos de politica e gestão ambiental: os caminhos do desenvolvimento sustentável. Rio de Janeiro: Garamond, 2012.

Chiapetti, R. J. N. \& Chiapetti, J. A água e os rios: imagens e imaginário da natureza. Geograficidade, 1(1), 71-90, 2011.

Dantas, C. E. O. Previsão e Controle de Inundações Em Meio Urbano Com Suporte de Informações Espaciais de Alta Resolução. Recife, Tese (Doutorado em Engenharia Civil) - UFPE, 2012.

Dash, A.K.; Bhattacharjee, R.M.; Paul, P.S. Lessons learnt from Indian inundation disasters: An analysis of case studies. International Journal of Disaster Risk Reduction, 20, 93-102, 2016.

Diniz, N.C. Cartografia geotécnica por classificação de unidades de terreno e avaliação de susceptibilidade e aptidão. Revista Brasileira de Geologia de Engenharia Ambiental, 2(1), 29-77, 2012.

Fiorillo, C. A. P. Estatuto da Cidade Comentado. São Paulo: Editora Revista dos Tribunais, 2002

Global Water Partnership-GWP. Towards Water Security: A Framework for Action. Estocolmo, Suécia e Londres (Reino Unido), 2000. Disponível em: <http://www.gwp. org/globalassets/global/toolbox/references/towards-water-security.-a-framework-for-action.-mobilising-political-will-to-act-gwp-2000.pdf>

Gonçalves, M. F. Previsão de chuva com auxílio de radar de tempo visando a um sistema de alerta antecipado de cheias em áreas urbanas. 2009. São Carlos, Dissertação (Mestrado em Hidráulica e Saneamento) - USP, 2009. Disponível em $<$ http://www.teses.usp.br/teses/disponiveis/18/18138/tde27042010-133924>

Guimarães, R. B; Guerreiro, J. A. S.; Peixoto, J. A. S. Considerações sobre os riscos ambientais e urbanos no tocante aos desastres e emergências. Revista VeraCidade, 3(3), 1-21, 2008.

Haigh, R.; Amaratunga, D. An integrative review of the built environment discipline's role in the development of society's resilience to disasters. International Journal of Disaster Resilience in the Built Environment, 1(1), 11-24, 2010.

Hakaloba, J. M.; Mumba, J.; Dambe, R.; Michelo, C. Examining the integration process of the community based institutions and organisations as a response strategy for 'disaster reduction': A case of the Kazungula District, Zambia. International Journal of Disaster Risk Reduction, 17, 273-279, 2016.

Isaranuwatchai, W.; Guerriere, D. N.; Andrews, G. J.; Coyte, P. C. Patterns of health service utilization following the Tsunami in Thailand. International Journal of Disaster Risk Reduction, 20, 14-18, 2016.

Jha, A.; Bloch, R.; Lamond, J. Cities and Flooding. A guide to integrated urban flood risk management for the 21st century. Washington: World Bank: 2012. DOI: 10.1596/9780-8213-8866-2

Jick, T. D. Mixing Qualitative and Quantitative Methods: Triangulation in Action. Administrative Science Quarterly, 24(4), 602-611, 1979.

Kazmierczak, A.; Cavan, G. Surface water flooding risk to urban communities: Analysis of vulnerability, hazard and exposure. Landscape and Urban Planning, 103 (2), 185-197, 2011.

Kobiyama, M.; Mendonça, M.; Moreno, D.A.; Marcelino, I. P. V. O.; Marcelino, E. V.; Gonçalves, E. F.; Brazetti, L. L. P.; Goerl, R. F.; Molleri, G. S. F.; Rudorff, F. M. Prevenção de desastres naturais: conceitos básicos. Curitiba: Organic Trading, 2006.

Lautza, J.; Manthrithilake, H. Water security: Old concepts, new package, what value? Natural Resources Forum, 36(2), 76-87, 2012.

Machado, M. L.; Nascimento, N. O.; Baptista, M. B.; Gonçalves, M.; Silva, A., Lima, J. C.; Dias, R.; Silva, A.; Machado, E.; Fernandes, W. Curvas de danos de inundação versus profundidade de submersão: desenvolvimento de metodologia. Revista de Gestão de Água da América Latina, 2(1), 35-52, 2005.

Manyena, S. B. Disaster event: window of opportunity to implement global disaster policies? Jàmbá: Journal of 
Disaster Risk Studies, 5(1), 1-10, 2013.

Marengo, J. A. Água e mudanças climáticas. Estudos Avançados, 22(63), 83-96, 2008.

Saito, C. H. Vulnerabilidades, riscos e desastres ambientais de menor exposição na mídia. In: SEABRA, G. (Org.). Educação ambiental: conceitos e aplicações. João Pessoa: UFPB, p. 227-239, 2013.

Sampaio, T. Q.; Pimentel, J.; Silva, C. R.; Moreira, H. F. A atuação do serviço geológico do Brasil (CPRM) na gestão de riscos e resposta a desastres naturais. In: Anais do VI Congresso CONSAD de Gestão Pública. Brasília-DF, 16-18 de abr., 2013. Disponível em: <http://www. escoladegestao.pr.gov.br/arquivos/File/2013/V_CONSAD/ VI_CONSAD/070.pdf>

Santin, J. R.; Marangon, E. G. O Estatuto da Cidade e os instrumentos de política urbana para proteção do patrimônio histórico: outorga onerosa e transferência do direito de construir. História, 27(2), 89-109, 2008.

Santos, I. A.; Berlinck, C. N.; Araújo, S. C. S.; Steinke, E. T.; Steinke, V. A.; Pianta, T. F.; Graebner, I. T.; Saito, C. H. The Centrality of the mediation concept in the participatory management of water. Canadian Journal of Environmental Education, 10(1), 180-194, 2005.

Santos, M. Metamorfoses do Espaço Habitado. 6. ed. São Paulo: Editora da Universidade de São Paulo. 2008.

Santos, I. A.; Saito, C. H. A mitificação da participação social na Política Nacional de Recursos Hidricos - gênese, motivação e inclusão social. Geosul, 21(42), 7-27, 2006.

Silva, J. A. Direito urbanístico brasileiro. 3. ed. São Paulo: Malheiros, 2000.
Toro, J. (coord.). Avaliação de perdas e danos: inundações bruscas em Pernambuco - Junho de 2010. Brasília: Banco Mundial, 2012a.

Toro, J. (coord.). Avaliação de perdas e danos: inundações bruscas em Alagoas - Junho de 2010. Brasília: Banco Mundial, 2012b.

Toro, J. (coord.). Avaliação de perdas e danos: inundações e deslizamentos na Região Serrana do Rio de Janeiro - Janeiro de 2011. Brasília: Banco Mundial, 2012c.

Toro, J. (coord.). Avaliação de perdas e danos: inundações bruscas em Santa Catarina - Novembro de 2008. Brasília: Banco Mundial, 2012d.

Toro, J.; Matera, M.; Moura, F. S.; Pedroso, F. F. Lidando com perdas: opções de proteção financeira contra desastres no Brasil. Washington: Banco Mundial, 2014. Disponível em: http://bibspi.planejamento.gov.br/bitstream/handle/iditem/658/Banco\%20Mundial_opcoes_de\%20proteção\%20 financeira $\% 20$ contra $\% 20$ desastres $\% 20$ no $\% 20$ Brasil. pdf? sequence $=1$

UNISDR. Hyogo framework for action 2005-2015: building the resilience of nations and communities to disasters. Geneva: UNISDR, 2007. Disponível em: < http://www. preventionweb.net/files/1217_HFAbrochureEnglish.pdf >

Valencio, N. Desastres, ordem social e planejamento em defesa civil: o contexto brasileiro. Saúde e Sociedade, 19(4), 748-762, 2010.

Zhou, L.; Perera, S.; Jayawickrama, J.; Adeniyi, O. The Implication of Hyogo Framework for Action for Disaster Resilience Education. Procedia Economics and Finance, 18, 576-583, 2014. 